RICYDE. Revista Internacional de Ciencias del Deporte doi: $10.5232 /$ ricyde

Rev. int. cienc. deporte

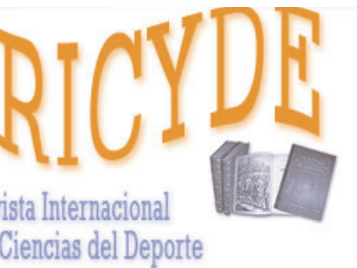

RICYDE. Revista Internacional de Ciencias del Deporte VOLUMEN XI - AÑO XI

Páginas:145-162 ISSN:1885-3137 No 40 - Abril - 2015

\title{
Efectos de un protocolo de entrenamiento de alta intensidad sobre marcadores fisiológicos de estrés en ratas \\ Physiological effects of the stress induced by a high-intensity exercise protocol in rats
}

\author{
Daniel Camiletti-Moirón, Gerardo Medina, Ángel Núñez, Ana Andrade, Rosario Martínez, Elena Nebot, \\ Garyfallia Kapravelou, Jesús M. Porres, María López-Jurado, Pilar Aranda, Virginia A. Aparicio
}

Departamento de Fisiología, Facultad de Farmacia y Facultad de Ciencias del Deporte, Instituto de Nutrición y Tecnología de los Alimentos e Instituto Mixto Universitario de Deporte y Salud. Universidad de Granada.

\section{Resumen}

El objetivo del presente estudio fue examinar los posibles efectos del estrés fisiológico producidos por un programa de entrenamiento de alta intensidad (EAI) en ratas. Cuarenta ratas Wistar fueron aleatoriamente distribuidas en 2 grupos experimentales: grupo EAI o grupo control, durante 12 semanas. Se estimaron índices biológicos, parámetros musculares, urinarios, plasmáticos, de perfil lipídico hepático y morfológicos renales. Tanto la ingesta media $(P<0.05)$ como el peso final y de la canal (ambos, $P<0,001)$ fueron menores en el grupo EAI. Los marcadores hormonales reflejaron mayores concentraciones de corticosterona $(P<0.05)$ junto con una disminución de las de testosterona $(P<0.05)$ en el grupo EAI, por lo que la ratio corticosterona/testosterona fue mayor y la de testosterona/corticosterona menor (ambas, $\mathrm{P}<0.01$ ). A nivel lipídico plasmático, el grupo EAI mostró menores concentraciones de triglicéridos $(P<0.01)$, pero también mayores de colesterol-LDL $(P<0.01)$. A nivel lipídico hepático, la cantidad de grasa fue mayor en el grupo EAI $(\mathrm{P}<0.01)$, aunque dicho grupo también presentó menores niveles de triglicéridos $(\mathrm{P}<0.05)$. El análisis de morfología renal mostró un aumento del tejido conectivo intersticial en el grupo de EAI $(P<0.05)$. El estrés inducido por el protocolo de EAI pudo conllevar un estado catabólico que podría haber anulado las esperadas ganancias musculares, alteró el perfil lipídico plasmático y hepático y un perfil renal con mayor predisposición a patologías futuras. El presente estudio trata de reproducir un modelo de estrés/sobreentrenamiento que podría darse en deportistas con la intención de profundizar algo más en el conocimiento del alcance que este estado fisiológico pudiera inducir a largo plazo en órganos poco estudiados y con ello, en la salud futura del deportista.

Palabras clave: estrés fisiológico; hipertrofia; riñón; metabolismo lipídico; ratas.

\section{Abstract}

The objective of this study was to examine the possible stress physiological effects of a high-intensity training (HIT) in rats. Forty Wistar rats were randomly divided into 2 experimental groups $(n=20)$ : EAI or control (sedentary) groups for 12 weeks. Biological, muscle, urinary, plasma, hepatic and renal markers were measured. Food intake $(P<0.05)$, final body weight and carcass weight (both, $P<0.001)$ were significantly lower in the HIT group. Hormonal markers reflected increased levels of corticosterone $(P<0.05)$ and decreased levels of testosterone $(\mathrm{P}<0.05)$ in the HIT group. Consequently, the ratio corticosterone/testosterone was higher and the testosterone/corticosterone lower in the HIT group (both, $\mathrm{P}<0.01$ ). Regarding hepatic lipid profile, the HIT group showed higher liver fat $(P<0.01)$ but lower hepatic triglycerides $(P<0.05)$. Regarding plasma lipid profile, the HIT group showed lower triglycerides concentration $(P<0.01)$ but also higher LDL cholesterol concentrations $(P<0.01)$. Finally, the renal morphology study showed increased kidney connective interstitial tissue $(P<0.05)$. The stress induced by the HIT protocol promoted a catabolism status that could lead to a lower muscular development, a worse hepatic and plasma lipid profile and a renal profile with increased susceptibility to future pathologies. The present study aimed to develop a stress/overtraining protocol that might be carried out in athletes with the purpose to deepen study the effects that this adverse status might induce in the long-term health of the athlete.

Key words: stress, physiological; hypertrophy; kidney; lipid metabolism; rats.

Correspondencia/correspondence: Daniel Camiletti-Moirón

Departamento de Fisiología, Facultad de Farmacia y Facultad de Ciencias del Deporte, Instituto de Nutrición y Tecnología de los Alimentos e Instituto Mixto Universitario de Deporte y Salud. Universidad de Granada. España

Email: dcamiletti@ugr.es 


\section{Introducción}

El estrés es un "síndrome de adaptación general", es decir, una sola respuesta estereotipada provocada por una demanda sobre el organismo (Rosmond, 2005). Los procesos catabólicos para la movilización de la energía y funciones anabólicas tales como el crecimiento o la sanación necesitan estar equilibrados para mantener la salud (Lundberg, 2005; McEwen, 2004). Factores de estrés psicológico y fisiológico pueden alterar las funciones neuroendocrinas, inmunológicas, de comportamiento y metabólicas (McEwen, 2004).

En la sociedad, entre las personas que practican deporte de competición, el estrés en formas aguda y crónica es frecuente (Meeusen, Duclos, Foster, Fry, Gleeson, Nieman, Raglin, Rietjens, Steinacker, y Urhausen, 2013; Rosmond, 2005). Para maximizar el rendimiento físico, atletas y entrenadores manipulan las cargas de entrenamiento a través de ajustes en duración, frecuencia e intensidad, o a través de una reducción del período de recuperación. Sin embargo, un desequilibrio acumulativo entre la carga de ejercicio y la recuperación puede inducir el denominado "síndrome de sobreentrenamiento" (Kreher y Schwartz, 2012; Meeusen y col., 2013). Dicho estado de sobreentrenamiento suele venir asociado a una disminución del rendimiento específico del deporte, fatigabilidad acelerada y sintomatología de estrés (Kreher y Schwartz, 2012; Meeusen y col., 2013). Entre las manifestaciones físicas y psicológicas que presentan los individuos afectados por el síndrome de sobreentrenamiento se encuentran la fatiga y debilidad muscular, pérdida de apetito y peso corporal, insomnio, depresión, ansiedad, dificultades para mantener la concentración, irritabilidad o cambios de humor (Kreher y Schwartz, 2012). Además, a nivel fisiopatológico, dicho síndrome podría debilitar el sistema inmune, alterar el perfil lipídico o favorecer la aparición de patologías cardiovasculares y renales, entre otras manifestaciones menos estudiadas (Czepluch, Barres, Caidahl, Olieslagers, Krook, Rickenlund, Zierath, y Waltenberger, 2011; Kreher y Schwartz, 2012; Lehmann, Wieland, y Gastmann, 1997; Wu, Chen, Huang, y Zhang, 2012).

El riesgo de que se presente un estado de sobreentrenamiento es mayor cuando se opta por la práctica de ejercicios de alta intensidad o con grandes volúmenes de trabajo (Kreher y Schwartz, 2012). La variabilidad individual en el potencial de recuperación, la capacidad de ejercicio, la tolerancia al estrés y la tolerancia al entrenamiento explica las diversas vulnerabilidades de atletas al sobreentrenamiento bajo estímulos de entrenamiento idénticos (Lehmann, Foster, y Keul, 1993). Además, las manifestaciones del sobreentrenamiento varían en base al perfil de la disciplina deportiva que desarrolle dicho deportista (Kreher y Schwartz, 2012; Meeusen y col., 2013). Así pues, en deportes donde predomina el metabolismo anaeróbico, como en velocistas o lanzadores, las alteraciones del estado de sobreentrenamiento son más de origen simpático (agitación, taquicardia, hipertensión, etc.), mientras que en las disciplinas en las que predomina el componente aeróbico, como puede ser la carrera de fondo o ciclismo de largas distancias, las manifestaciones son más de tipo parasimpático (bradicardia, fatiga, depresión, etc.) (Kreher y Schwartz, 2012; Meeusen y col., 2013).

A pesar de su alta prevalencia y riesgo potencial para la salud del deportista, las manifestaciones fisiológicas y morfológicas del estado de sobreentrenamiento sobre ciertos órganos y sistemas no se conocen en profundidad, ni bajo diseños experimentales bien controlados (Kreher y Schwartz, 2012). En este sentido, modelos experimentales animales están ganando peso a la hora de estudiar y reproducir los efectos fisiopatológicos del estrés inducido por el sobreentrenamiento (Kreher y Schwartz, 2012; Pereira, Filho, Alves, Pauli, Ropelle, Souza, Cintra, Saad, y Silva, 2012; Xiao, Chen, y Dong, 2012). De hecho, algunos autores han diseñado y publicado modelos experimentales animales de estrés basados en protocolos de ejercicio físico (Pereira y col., 2012). 
Por lo tanto, en el presente estudio hemos tratado de inducir un estado de sobreentrenamiento en ratas a partir de un programa de EAI con la intención de valorar los efectos que el sobreentrenamiento pudiera tener sobre órganos y sistemas sobre los que los efectos de dicho estado fisiológico han sido poco estudiados. Los objetivos de esta investigación fueron: i. contrastar, mediante índices biológicos, musculares y hormonales, que un protocolo de EAI, basado en el desarrollo de la fuerza hipertrofia, puede inducir un estado de sobreentrenamiento en ratas; ii, analizar los efectos de dicho sobreentrenamiento sobre órganos y sistemas poco estudiados (hígado y riñón, principalmente).

\section{Material y método}

\section{Animales y diseño experimental}

Para el presente estudio, un total de 40 ratas Wistar macho albinas fueron distribuidas aleatoriamente en 2 grupos experimentales: grupo de ratas sedentarias $(n=20)$ y grupo sometido a EAI $(n=20)$. Los animales, de 8 semanas de edad y con un peso inicial de $165 \pm 8$ $\mathrm{g}$, fueron alojados desde el primer día del periodo de adaptación (una semana antes del comienzo del periodo experimental) en jaulas individuales de metabolismo diseñadas para la recogida por separado de heces y orina. Las jaulas estaban ubicadas en una habitación bien ventilada, termorregulada $\left(21 \pm 2^{\circ} \mathrm{C}\right)$, con un rango de humedad relativa de entre el 40 y el $60 \%$ y un ciclo inverso de luz de 12:12 horas (08:00-20:00 h). Durante todo el periodo experimental (90 días) los animales tuvieron pleno acceso al agua destilada, y consumieron la dieta experimental ad libitum.

\section{Desarrollo de los experimentos}

El peso de los animales fue registrado todas las semanas a la misma hora del día (08:00 de la mañana), tras 12 horas de ayuno. Así mismo, la cantidad de dieta diaria consumida por cada uno de los animales fue cuantificada. Se formuló una dieta normocalórica en base a las recomendaciones nutricionales de rata de laboratorio del Colegio Americano de Nutrición (AIN-93) (Reeves, Nielsen, y Fahey, 1993). La composición básica de las dietas experimentales es la AIN-93M, con un $10 \%$ de proteína. Hemos empleado hidrolizados proteicos comerciales de soja y lactosuero como únicas fuentes de proteína, ya que están ampliamente disponibles en el mercado y se consumen frecuentemente por los deportistas que acuden a gimnasios con la intención de lograr ganancias musculares.

A los 89 días del periodo experimental, una muestra de 12 horas de orina de cada animal fue recogida para su análisis bioquímico. La orina fue posteriormente pesada y transferida a tubos graduados para el análisis del $\mathrm{pH}$.

Al final del periodo experimental los animales fueron anestesiados con ketamina-xilacina desangrados mediante canulación de la arteria aorta a nivel abdominal. La sangre fue extraída (con heparina como anticoagulante) y centrifugada a $4500 \mathrm{rpm}$ durante 15 minutos para separar el plasma, que fue congelado en Nitrógeno $(\mathrm{N})$ líquido y conservado a $-80^{\circ} \mathrm{C}$ para el posterior análisis bioquímico. Los músculos gastronemios fueron extraídos para el análisis de N. Los riñones fueron extraídos, pesados y el izquierdo conservado en formalina para su posterior análisis histológico. Para analizar el efecto del EAI sobre la grasa y la masa muscular, que pudieran verse afectadas por el entrenamiento (posibles ganancias de masa muscular y pérdida de masa grasa), se cuantificó el peso de la canal del animal. La canal corresponde al peso del animal tras ser sacrificado y desangrado, sin piel, grasa, cabeza, extremidades ni vísceras. Por último, la extracción de la grasa del hígado se llevó a cabo mediante el método de Folch, con ligeras adaptaciones (Folch, Lees, y Sloane Stanley, 1957). 
Todos los experimentos fueron desarrollados siguiendo las Normas de Ética en Investigación en Ciencias del Deporte (Harriss y Atkinson, 2011) y de acuerdo a la Guía Europea de Alojamiento y Cuidado Animal (European Community Council, 1986) (EstoppeyStojanovski, 1986). Además, todos los procedimientos que se han llevado a cabo en el presente estudio han sido aprobados por el Comité Ético de Experimentación Animal de la Universidad de Granada.

\section{Entrenamiento de los animales}

El grupo experimental fue entrenado siguiendo un protocolo de entrenamiento de alta intensidad basado en el desarrollo de la fuerza hipertrofia en un tapiz rodante de diseño especial para ratas (Panlab de 5 calles, LE8710R), con cargas regulables en una bolsa atada con una cuerda a la cola del animal. Este tipo de entrenamiento se eligió con el fin de reproducir el tipo de ejercicio realizado por personas interesadas en ganar masa y fuerza muscular. Por lo tanto, dicho protocolo sigue los principios básicos del entrenamiento para el desarrollo de la fuerza hipertrofia en humanos, implicando incrementos de cargas, repeticiones y series para maximizar las ganancias musculares (de Salles, Simao, Miranda, Novaes Jda, Lemos, y Willardson, 2009).

El grupo de entrenamiento se ejercitó en días alternos (3-4 sesiones/semana). Los animales corrieron a una velocidad constante de $38 \mathrm{~cm} / \mathrm{s}$ durante todo el período experimental en su ciclo de oscuridad (de activación). Antes de iniciar el periodo experimental, los animales fueron adaptados al tapiz durante una semana, los primeros tres días sin peso, y los últimos cuatro días arrastrando el $20 \%$ de su peso corporal. Dicho entrenamiento finalizó cuatro días previos al fin del periodo experimental con la intención de no interferir de forma aguda sobre ciertos parámetros plasmáticos (por ejemplo de las concentraciones de creatina quinasa).

El número de sesiones llevadas a cabo cada semana, el número de series por sesión y el tiempo empleado en cada sesión en conjunto, así como la carga soportada por los animales, se muestran en la Tabla 1.

Tabla 1. Descripción del protocolo de entrenamiento de alta intensidad

\begin{tabular}{ccccc}
\hline Semana & $\begin{array}{c}\text { Tiempo de } \\
\text { trabajo }(\mathbf{m i n})\end{array}$ & Series & $\begin{array}{c}\text { Descanso entre series } \\
(\mathbf{m i n})\end{array}$ & $\begin{array}{c}\text { Carga } \\
\text { (\% 1 RM) }\end{array}$ \\
\hline 1 & 2 & 10 & 1 & 55 \\
2 & 2 & 10 & 1 & 60 \\
3 & 2 & 10 & 1 & 65 \\
4 & 2 & 10 & 1,5 & 70 \\
5 & 2 & 10 & 1,5 & 70 \\
6 & 2,5 & 10 & 1,5 & 75 \\
7 & 2,5 & 12 & 1,5 & 75 \\
8 & 2 & 12 & 2 & 80 \\
9 & 2,5 & 12 & 2 & 80 \\
10 & 1,5 & 12 & 2 & 85 \\
11 & 2 & 12 & 2,5 & 85 \\
12 & 1 & 12 & 2,5 & 85 \\
\hline
\end{tabular}

RM, Repetición máxima 
Desde la primera semana del período experimental hasta la finalización del estudio, los pesos de la bolsa (cargas) se incrementaron progresivamente y se ajustaron individualmente una vez por semana en base al porcentaje de una repetición máxima (1 RM), que se define como la carga máxima que la rata podía arrastrar en su bolsa. La prueba de estimación de 1RM se realizó como se describe a continuación: el animal es colocado en una superficie plana, horizontal y no resbaladiza con la bolsa atada a su cola con una carga específica. El animal es estimulado acústicamente e inmediatamente reacciona desplazándose. Este procedimiento se repite varias veces, aumentando la carga cada vez, hasta que la carga es tan pesada que la rata no puede avanzar con ella aún siendo estimulada acústicamente. La carga alcanzada en este punto se considera 1RM.

Los animales sedentarios fueron manipulados de forma exactamente igual a los grupos de entrenamiento, exceptuando el propio entrenamiento, con el fin de evitar un efecto contaminante debido al contacto humano.

\section{Análisis químicos}

El contenido total de $\mathrm{N}$ del suplemento proteico y del músculo gastronemio se determinó por el método de Kjeldahl. La proteína cruda se calculó como $\mathrm{N}$ x 6,25.

$\mathrm{El} \mathrm{pH}$ de la orina fue analizado con un pH-metro (Crison, Barcelona, España).

Las concentraciones plasmáticas de colesterol total, colesterol LDL y HDL, triglicéridos, hierro, lactato deshidrogenasa (LDH) y creatina quinasa (CK) se midieron con un autoanalizador (Hitachi-p800 Roche, F. Hoffmann-La Roche Ltd. Suiza). Para la determinación del colesterol y triglicéridos hepáticos se utilizaron kits comerciales (Spinreact, S.A. Gerona, España). 
Las concentraciones de corticosterona en plasma se midieron por radioinmunoensayo utilizando un kit comercial (CorticosteronaI-125Kitpara rata/ratón; DRG International Inc. EE.UU). Todas las muestras se analizaron por duplicado y en el mismo ensayo. El coeficiente de variación intraensayo fue del 4,4\% y la sensibilidad fue de 7,7 ng/ml. Las concentraciones de testosterona en plasma también se estimaron por duplicado y mediante radioinmunoensayo utilizando un kit comercial (TESTO-CTK I-125Kit; DiaSorin, Italia). El coeficiente de variación intraensayo fue del 5,1\% y la sensibilidad fue de $0,02 \mathrm{ng} / \mathrm{ml}$. Adicionalmente, los índices corticosterona/testosterona y testosterona/corticosterona fueron calculados.

\section{Análisis morfológico renal}

Las muestras del riñón izquierdo fueron fijadas en formalina tamponada al $4 \%$ y se introdujeron en parafina. Posteriormente, se obtuvieron secciones de cuatro micrómetros de espesor que fueron teñidas con picro-sirius1\% F3BA rojo (Gurr, BDH Chemicales Ltd, Poole, Reino Unido) (Sweat, Puchtler, y Rosenthal, 1964). Esta técnica permite la visualización de fibras conectivas de color rojo oscuro sobre un fondo amarillo pálido (Sweat, Puchtler, y Rosenthal, 1964).Las secciones fueron evaluadas por microscopía óptica. Cuarenta imágenes por muestra fueron capturadas: veinte de los glomérulos para determinar la morfometría y el tejido conectivo intraglomerular y veinte del área tubulointersticial para medir el tejido conectivo intersticial. Todas las imágenes fueron adquiridas con objetivo 20x y analizadas con el software FibrosisHR ${ }^{\circledR}$ (Masseroli, O'Valle, Andujar, Ramirez, Gomez-Morales, de Dios Luna, Aguilar, Aguilar, Rodriguez-Puyol, y Del Moral, 1998). Esta aplicación de análisis de imágenes nos permite cuantificar de forma automática los parámetros morfométricos usando varios algoritmos de procesamiento de imágenes (Masseroli y col., 1998).

Se estimaron las siguientes variables: a) Porcentaje de tejido conectivo intersticial en referencia al área de la imagen, excluyendo el área glomerular (el tejido conectivo que hay en el intersticio más la capsula de Bowman). b) El área de tejido conectivo intersticial incluyendo la capsula de Bowman (sin glomérulo). c) El área glomerular.

\section{Tratamiento estadístico de los datos}

Los resultados se presentan como media y desviación típica, a no ser que se indique lo contrario. Los efectos del EAI sobre las distintas variables incluidas en el estudio en comparación con el grupo sedentario fueron analizados mediante un análisis de la varianza (ANOVA). Todos los análisis se llevaron a cabo con el software estadístico SPSS, versión 19.0 para Windows (SPSS Inc., Chicago, IL), y el nivel de significación se estableció en P $<0,05$.

\section{Resultados}

La cantidad ingerida de alimento, el peso corporal y parámetros musculares, así como los marcadores hormonales de estrés, se muestran en la Tabla 2. 
Camiletti-Moirón, D.; Medina, G.; Nuñez, A.; Andrade, A.; Nartínez, R.; Nebot, E.; Kapravelou, G.; Porres, J.M.; López-Jurado, M.; Aranda, P., Aparicio, V. (2015). Efectos de un protocolo de entrenamiento de alta intensidad sobre marcadores fisiológicos de estrés en ratas. RICYDE. Revista internacional de ciencias del deporte, 40(11), 145-162. http://dx.doi.org/10.5232/ricyde2015.04004

Tabla 2. Efectos del entrenamiento de alta intensidad sobre el peso, la ingesta y parámetros musculares y hormonales

\begin{tabular}{lcc}
\hline & Sedentario & $\begin{array}{c}\text { Entrenamiento de alta } \\
\text { intensidad }\end{array}$ \\
\hline Ingesta media (g/día) & $17,07 \pm 2,35$ & $14,89 * \pm 1,76$ \\
Peso final (g) & $340,63 \pm 33,75$ & $310,56 * * * \pm 35,58$ \\
Canal (g) & $176,49 \pm 17,76$ & $164,88^{* * *} \pm 18,10$ \\
Nitrógeno gastronemio (g/100g SS) & $13,47 \pm 0,82$ & $13,92 \pm 0,76$ \\
\hline Marcadores hormonales de estrés & & \\
\hline Corticosterona (nmol/L) & & $28,05 * * \pm 4,56$ \\
Testosterona (nmol/L) & $23,30 \pm 6,16$ & $2,96 * \pm 2,54$ \\
Ratio corticosterona/testosterona & & $0,20 * * \pm 0,14$ \\
& $4,72 \pm 3,39$ & \\
\hline
\end{tabular}

Valores mostrados como media \pm desviación típica. $* \mathrm{P}<0,05, * * \mathrm{P}<0,01$ y $* * * \mathrm{P}<0,001 ; \mathrm{SS}$, sustancia seca.

La ingesta media de los animales entrenados fue significativamente menor en comparación con el grupo sedentario ( $\mathrm{P}=0,021)$, así como los valores de peso final y de la canal (ambos, $\mathrm{P}<0,001)$. A nivel muscular encontramos una ligeramente mayor, aunque no significativa, fijación de $\mathrm{N}$ en el músculo gastronemio $(\mathrm{P}=0,062)$.

Se observaron mayores concentraciones de corticosterona $(\mathrm{P}=0,029)$ y de la ratio corticosterona/testosterona $(\mathrm{P}=0,006)$; junto con una disminución de los niveles de testosterona $(\mathrm{P}=0,029)$ y de la ratio testosterona/corticosterona $(\mathrm{P}=0,002)$ en el grupo de EAI en comparación con el sedentario.

Los marcadores de perfil lipídico plasmático y hepático se muestran en la Tabla 3. 
Camiletti-Moirón, D.; Medina, G.; Nuñez, A.; Andrade, A.; Nartínez, R.; Nebot, E.; Kapravelou, G.; Porres, J.M.; López-Jurado, M.; Aranda, P., Aparicio, V. (2015). Efectos de un protocolo de entrenamiento de alta intensidad sobre marcadores fisiológicos de estrés en ratas. RICYDE. Revista internacional de ciencias del deporte, 40(11), 145-162. http://dx.doi.org/10.5232/ricyde2015.04004

Tabla 3. Efectos del entrenamiento de alta intensidad sobre el perfil lipídico plasmático y hepático

\begin{tabular}{|c|c|c|}
\hline & Sedentario & Entrenamiento de alta intensidad \\
\hline \multicolumn{3}{|l|}{ Perfil lipídico plasmático } \\
\hline Colesterol total (mg/dl) & $46,78 \pm 11,89$ & $44,63 \pm 13,11$ \\
\hline Triglicéridos (mg/dl) & $81,23 \pm 72,18$ & $54,28 * * \pm 32,49$ \\
\hline Colesterol-HDL (mg/dl) & $19,42 \pm 11,97$ & $22,88 \pm 13,56$ \\
\hline Colesterol-LDL (mg/dl) & $5,54 \pm 4,35$ & $8,08 * * \pm 5,36$ \\
\hline \multicolumn{3}{|l|}{ Perfil lipídico hepático } \\
\hline Grasa (g) & $0,025 \pm 0,008$ & $0,030 * * \pm 0,01$ \\
\hline Grasa (\%) & $6,83 \pm 2,00$ & $7,88 * * \pm 2,47$ \\
\hline Colesterol (mg/g) & $3,78 \pm 1,17$ & $3,51 \pm 1,29$ \\
\hline Triglicéridos (mg/g) & $3,67 \pm 2,19$ & $2,99 * \pm 1,25$ \\
\hline
\end{tabular}

Valores mostrados como media \pm desviación típica. $* \mathrm{P}<0.05, * * \mathrm{P}<0.01$ y $* * * \mathrm{P}<0.001$. HDL, lipoproteína de alta densidad; LDL, lipoproteína de baja densidad

A nivel lipídico plasmático, el grupo EAI mostró menores concentraciones de triglicéridos con respecto al control $(\mathrm{P}=0,004)$, pero mayores concentraciones de colesterol LDL $(\mathrm{P}=0,002)$, sin diferencias en colesterol total ni colesterol HDL. A nivel lipídico hepático, se aprecian diferencias en cuanto a la cantidad de grasa en hígado, siendo más elevada en el grupo EAI $(\mathrm{P}<0,01)$. Por el contrario, el grupo EAI presentó menores niveles de triglicéridos hepáticos $(\mathrm{P}=0,022)$ y no se apreciaron diferencias en las concentraciones de colesterol total.

Otros valores plasmáticos y urinarios relacionados con el EAI, así como la morfología renal se muestran en la Tabla 4. 
Camiletti-Moirón, D.; Medina, G.; Nuñez, A.; Andrade, A.; Nartínez, R.; Nebot, E.; Kapravelou, G.; Porres, J.M.; López-Jurado, M.; Aranda, P., Aparicio, V. (2015). Efectos de un protocolo de entrenamiento de alta intensidad sobre marcadores fisiológicos de estrés en ratas. RICYDE. Revista internacional de ciencias del deporte, 40(11), 145-162. http://dx.doi.org/10.5232/ricyde2015.04004

Tabla 4. Efectos del entrenamiento de alta intensidad sobre marcadores plasmáticos, urinarios y de morfología renal.

\begin{tabular}{|c|c|c|}
\hline & Sedentario & $\begin{array}{c}\text { Entrenamiento de alta } \\
\text { intensidad }\end{array}$ \\
\hline Creatina Quinasa (UI/L) & $1267,7 \pm 1115,2$ & $2039,5 * \pm 1816,0$ \\
\hline Lactato deshidrogenasa (UI/L) & $1615,3 \pm 1539,0$ & $1678,0 \pm 1444,3$ \\
\hline Hierro (mg/dl) & $194,03 \pm 114,4$ & $146,75^{* *} \pm 58,3$ \\
\hline pH urinario & $6,62 \pm 0,57$ & $6,37 * \pm 0,46$ \\
\hline \multicolumn{3}{|l|}{ Parámetros renales } \\
\hline Riñón (g) & $1,09 \pm 0,23$ & $1,00 \pm 0,18$ \\
\hline Riñón (g/100g peso) & $0,32 \pm 0,06$ & $0,31 \pm 0,04$ \\
\hline Tejido conectivo intersticial (\%) & $3,02 \pm 1,05$ & $3,81 * \pm 1,20$ \\
\hline Tejido conectivo intersticial $\left(\mu \mathrm{m}^{2}\right)$ & $3951 \pm 1380$ & $5021 * \pm 1631$ \\
\hline Área glomerular $\left(\mu m^{2}\right)$ & $43497 \pm 4974$ & $42855 \pm 4809$ \\
\hline
\end{tabular}

Valores mostrados como media \pm desviación típica. $* \mathrm{P}<0.05, * * \mathrm{P}<0.01 \mathrm{y} * * * \mathrm{P}<0.001$.

Los niveles plasmáticos de $\mathrm{CK}$ fueron superiores en el grupo $\mathrm{EAI}(\mathrm{P}=0,011)$, a la vez que dicho grupo mostró una menor concentración plasmática de hierro $(\mathrm{P}=0,002)$. El pH urinario fue ligeramente más ácido en el grupo de $\mathrm{EAI}(\mathrm{P}=0,046)$. No se observaron diferencias en las concentraciones plasmáticas de LDH.

El grupo EAI presentó un aumento del tejido conectivo intersticial renal en porcentaje y área (ambos, $\mathrm{P}=0,03$ ). No se observaron diferencias en el peso del riñón ni en el área glomerular.

\section{Discusión}

Los principales hallazgos de este estudio parecen confirmar los posibles efectos adversos a nivel lipídico, renal y hepático del estrés inducido por un protocolo de EAI en ratas. Hemos confirmado el estado de sobreentrenamiento en el grupo EAI a través de la disminución de la ingesta, la pérdida de peso, las casi inexistentes ganancias musculares, las menores concentraciones plasmáticas de hierro y los índices hormonales de estrés (aumento de la corticosterona, reducción de la testosterona y alteración de las ratios corticosterona: testosterona y testosterona:corticosterona) (Adlercreutz, Harkonen, Kuoppasalmi, Naveri, Huhtaniemi, Tikkanen, Remes, Dessypris, y Karvonen, 1986; Liu, He, y Li, 2013; Meeusen y 
Camiletti-Moirón, D.; Medina, G.; Nuñez, A.; Andrade, A.; Nartínez, R.; Nebot, E.; Kapravelou, G.; Porres, J.M.; López-Jurado, M.; Aranda, P., Aparicio, V. (2015). Efectos de un protocolo de entrenamiento de alta intensidad sobre marcadores fisiológicos de estrés en ratas. RICYDE. Revista internacional de ciencias del deporte, 40(11), 145-162. http://dx.doi.org/10.5232/ricyde2015.04004

col., 2013; Priya y Reddy, 2012; Smith, Ben-Shlomo, Beswick, Yarnell, Lightman, y Elwood, 2005; Urhausen, Gabriel, y Kindermann, 1995; Wang, Wang, Chen, Lin, Gao, y Zhang, 2012). A pesar de la pérdida de peso, el grupo EAI mostró un perfil lipídico hepático y plasmático menos favorable, con mayor cantidad de grasa en hígado y mayores concentraciones plasmáticas de colesterol LDL. Además, los riñones de los animales con posible estado de sobreentrenamiento presentaron una peor morfología.

El cortisol es un glucocorticoide liberado por la corteza adrenal en respuesta al estrés que podría jugar un papel importante en la remodelación del tejido en respuesta al ejercicio intenso (Fragala, Kraemer, Denegar, Maresh, Mastro, y Volek, 2011; Kraemer, Fleck, Callister, Shealy, Dudley, Maresh, Marchitelli, Cruthirds, Murray, y Falkel, 1989; Kraemer, Noble, Clark, y Culver, 1987; Kraemer y Ratamess, 2005). En modelos experimentales animales, el aumento de las concentraciones de corticosterona (equivalente al cortisol en humanos), tiene como efecto la inhibición de la secreción de testosterona (Smith y col., 2005). Además, el estrés induce un deterioro de las células de Leydig, así como de la espermatogénesis en ratas (Priya y Reddy, 2012; Wang y col., 2012). La importancia de la secreción de la testosterona en el organismo viene marcada por el papel clave que desempeña a la hora de conseguir la recuperación de las fibras musculares, las ganancias de la fuerza y masa muscular y el incremento de la densidad y resistencia ósea (Xiao, Chen, y Dong, 2012). El síndrome de sobreentrenamiento se ha relacionado con la insuficiencia suprarrenal. De hecho, existe una relación directa entre el estrés físico crónico y la pérdida de funcionalidad de las glándulas suprarrenales, por lo que el rendimiento deportivo se vería gravemente afectado (Brooks y Carter, 2013). Por estos motivos, la ratio testosterona:cortisol se emplea como una medida del equilibrio anabólico y catabólico (Filaire, Bernain, Sagnol, y Lac, 2001; Urhausen, Gabriel, y Kindermann, 1995), estableciéndose que una disminución superior al 30\% puede ser indicativa de un estado de sobreentrenamiento (Adlercreutz y col., 1986; Filaire, Bernain, Sagnol, y Lac, 2001). Nuestros resultados han confirmado estos hallazgos observando mayores niveles de corticosterona y menores de testosterona en plasma en el grupo de EAI, lo que ha derivado en una reducción del 52\% de la ratio testosterona:cortisol. No obstante, la liberación de cortisol en relación a la testosterona podría variar en función a la naturaleza del ejercicio realizado (Slivka, Hailes, Cuddy, y Ruby, 2010), y ciertos autores no han confirmado un incremento de la ratio cortisol:testosterona ante entrenamientos de fuerza de alta intensidad (Fry, Kraemer y Ramsey, 1998).

El entrenamiento de fuerza puede tener efectos metabólicos importantes, tales como la reducción de la masa grasa y el colesterol LDL y los triglicéridos, con la consiguiente reducción del riesgo de enfermedad cardiovascular (Houston, Fazio, Chilton, Wise, Jones, Barringer, y Bramlet, 2009). Un protocolo de entrenamiento con cargas diseñado para la ganancia de masa muscular, como el desarrollado en nuestro estudio, se espera que provoque ganancias a nivel estructural-plástico, como así se ha confirmado en otros estudios donde se han aplicado programas de entrenamiento a intensidades moderadas en ratas. Sirva como ejemplo el de Matsakas, Macharia y col. (2012), en el que 14 animales divididos en tres grupos (ejercicio en rueda, ejercicio en agua y grupo de ratas sedentarias) se sometieron a 5 semanas de entrenamiento resistido, observándose un aumento significativo de masa muscular en ambos grupos de entreno con respecto al grupo control. Sin embargo, los resultados obtenidos en nuestra muestra con un protocolo de EAI no reflejaron una clara ganancia de masa muscular, lo que podría explicarse por la menor concentración de testosterona (estado catabólico) observada en dicho grupo, y de ahí que la adherencia de $\mathrm{N}$ (proteína) al músculo no haya sido notable. Asimismo, también hemos observado mayores concentraciones de CK 
Camiletti-Moirón, D.; Medina, G.; Nuñez, A.; Andrade, A.; Nartínez, R.; Nebot, E.; Kapravelou, G.; Porres, J.M.; López-Jurado, M.; Aranda, P., Aparicio, V. (2015). Efectos de un protocolo de entrenamiento de alta intensidad sobre marcadores fisiológicos de estrés en ratas. RICYDE. Revista internacional de ciencias del deporte, 40(11), 145-162. http://dx.doi.org/10.5232/ricyde2015.04004

en el grupo EAI, lo que podría ser indicativo de cierto grado de distrofia muscular (Oudman, Clark, y Brewster, 2013). Inicialmente, nuestro grupo pretendía diseñar un protocolo de entrenamiento basado en altas cargas con fines de ganancias de hipertrofia muscular, pero en este caso, dicho objetivo no se ha cumplido por el estrés derivado en el animal tras el desarrollo de dicho protocolo de ejercicio a altas intensidades. Por lo tanto, confirmamos que el sobreentrenamiento podría comprometer el rendimiento físico del deportista a través de un peor estado muscular, entre otros factores (Oudman, Clark, y Brewster, 2013; Xiao, Chen y Dong, 2012).

A diferencia de lo descrito en otros estudios desarrollados en ratas con protocolos de ejercicio a intensidad moderada (Ishigaki, Koyama, Tsujita, Tanaka, Hori, y Oku, 2005; Lira, Rosa, Pimentel, Tarini, Arida, Faloppa, Alves, do Nascimento, Oyama, Seelaender, de Mello, y Santos, 2010), hemos detectado valores adversos en algunos marcadores de perfil lipídico en el grupo de EAI, como mayor colesterol LDL plasmático y mayores concentraciones de grasa en hígado. Tan solo los triglicéridos, presumiblemente por ser la principal fuente energética en el ejercicio, han sido menores en el grupo de ratas de EAI con respecto a las sedentarias. Este fenómeno podría atribuirse a una posible alteración metabólica provocada por el estrés (Catalina-Romero, Calvo, Sanchez-Chaparro, Valdivielso, Sainz, Cabrera, GonzalezQuintela, y Roman, 2013; Depke, Fusch, Domanska, Geffers, Volker, Schuett, y Kiank, 2008). De hecho, en humanos se ha observado una clara asociación entre estrés laboral y dislipidemias (Catalina-Romero y col., 2013). En el estudio de Catalina-Romero y col. (2013), tras ajustar por posibles factores de confusión, el estrés laboral se asoció con el diagnóstico positivo de dislipidemia, colesterol LDL elevado y colesterol HDL bajo. En deportistas, y en concordancia con nuestros hallazgos, Ruiz y col. (2004) observaron que el ejercicio físico que induce un alto nivel de estrés puede conducir a alteraciones en el perfil lipídico plasmático. Para ello se estudió a un grupo de 28 nadadores, 17 jugadores de voleibol y 23 jugadores de fútbol, y un grupo control de 26 sujetos sanos sedentarios. Se observó que las personas que practican deportes que implican un alto nivel de esfuerzo físico (los jugadores de voleibol y fútbol) tenían un perfil lipídico menos favorable en comparación con los sujetos control. En la misma línea, en el estudio realizado por Martin-Cordero y col. (2011) 36 ratas se dividieron en 4 grupos: ratas sedentarias $(n=9)$, ratas sedentarias que realizaron una única sesión de ejercicio extenuante $(n=9)$, ratas entrenadas $(n=9)$ y ratas entrenadas que llevaron a cabo una sola sesión de ejercicio agudo $(n=9)$, durante 14 semanas. Los autores concluyeron que una intensidad de ejercicio inadecuada puede empeorar la regulación hormonal, lo que contribuye a los trastornos metabólicos, inflamatorios y de estrés asociados con el síndrome metabólico. Finalmente, hasta donde nuestro conocimiento nos lo permite, ningún estudio ha analizado los efectos del estrés sobre el perfil lipídico hepático. En nuestro diseño experimental las ratas sometidas a EAI presentaron mayor porcentaje graso en hígado, aunque menor concentración de triglicéridos.

En la población general, el ejercicio parece mejorar el estado renal (Moinuddin y Leehey, 2008; Peng, Chen, Hsieh, y Peng, 2012; Peng, Chen, Lu, y Peng, 2012; Pinheiro-Mulder, Aguila, Bregman, y Mandarim-de-Lacerda, 2010; Poortmans y Ouchinsky, 2006). De hecho, la práctica regular de ejercicio físico moderado podría mejorar la ratio de filtración glomerular 
Camiletti-Moirón, D.; Medina, G.; Nuñez, A.; Andrade, A.; Nartínez, R.; Nebot, E.; Kapravelou, G.; Porres, J.M.; López-Jurado, M.; Aranda, P., Aparicio, V. (2015). Efectos de un protocolo de entrenamiento de alta intensidad sobre marcadores fisiológicos de estrés en ratas. RICYDE. Revista internacional de ciencias del deporte, 40(11), 145-162. http://dx.doi.org/10.5232/ricyde2015.04004

y controlar la microalbuminuria (Moinuddin y Leehey, 2008; Pinheiro-Mulder, Aguila, Bregman, y Mandarim-de-Lacerda, 2010; Poortmans y Ouchinsky, 2006). El ejercicio también puede disminuir la inflamación renal (Pinheiro-Mulder, Aguila, Bregman, y Mandarim-de-Lacerda, 2010). En el presente estudio hemos observado un menor, pero no significativo, peso del riñón en los animales entrenados, lo que podría significar una menor inflamación renal o una menor hipertrofia renal. Estos resultados concuerdan con los obtenidos por nuestro grupo en un estudio previo con similar protocolo de entrenamiento, donde los animales entrenados también mostraron un menor peso del riñón (Aparicio, Nebot, Kapravelou, Sanchez, Porres, Lopez Jurado, y Aranda, 2011). Sin embargo, el análisis histológico mostró mayor tejido conectivo intersticial en el grupo de EAI, el cual es considerado precursor de fibrosis y por lo tanto podría predisponer a patologías renales a largo plazo. De hecho, un estudio previo analizó únicamente los efectos del EAI sobre la morfología renal, obteniendo un peor perfil morfológico tras el desarrollo de este protocolo de este protocolo de entrenamiento, y consecuentemente un mayor riesgo de enfermedades renales a largo plazo (Aparicio, Tassi, Nebot, Camiletti-Moiron, Ortega, Porres, y Aranda, 2014).

Algunas hipótesis podrían justificar este peor perfil renal fruto del sobreentrenamiento. i. El ejercicio extenuante o de alta intensidad puede provocar daño muscular que deriva en la liberación de proteínas musculares tales como la $\mathrm{CK}$, LDH, o mioglobina (Skenderi, Kavouras, Anastasiou, Yiannakouris, y Matalas, 2006; Warren, Blumbergs, y Thompson, 2002). La función renal podría verse alterada cuando la concentración de dichas moléculas se incrementa (Holt y Moore, 2000). ii. El cortisol también podría jugar un papel importante en la remodelación del tejido renal en respuesta al ejercicio intenso (Fragala y col., 2011; Kraemer y col., 1989; Kraemer, Noble, Clark, y Culver, 1987; Kraemer y Ratamess, 2005). Mantener los niveles de corticosterona en cantidades suprafisiológicas puede dar lugar a la modificación de la estructura y de la función renal (Benghuzzi, Tucci, Hughes, Lyon, y Adams, 2005). iii. Como se ha mencionado anteriormente, el grupo de EAI también presentó unas concentraciones $60 \%$ menores de testosterona en comparación con el grupo sedentario. La disfunción gonadal es frecuente en los hombres con enfermedad renal crónica y la deficiencia de testosterona está presente entre el 26-66\% de los hombres con diferentes grados de insuficiencia renal (Iglesias, Carrero, y Diez, 2012). Datos experimentales y clínicos sugieren que la testosterona podría tener implicaciones clínicas importantes con respecto a la progresión de la enfermedad renal (Iglesias, Carrero, y Diez, 2012). iv. Finalmente, el EAI, como es el de fuerza hipertrofia, puede provocar hipoxia, hipoglucemia o estrés oxidativo, situaciones que podrían inducir estrés del retículo endoplásmico, induciendo daño renal a nivel glomerular y tubular (Dickhout y Krepinsky, 2009; Inagi, 2009). En línea con estos resultados, recientemente algunos autores han encontrado una asociación entre sobreentrenamiento y estrés oxidativo (Liu, He, y Li, 2013).

\section{Limitaciones}

El presente estudio tiene algunas limitaciones que deben ser mencionadas. En primer lugar, los resultados fisiológicos obtenidos en roedores deben ser confirmados en humanos. Dicho con otras palabras, los efectos observados tras tres meses de EAI en ratas no pueden 
extrapolarse directamente a los posibles efectos en humanos dado que éstos tienen una motivación intrínseca hacia la práctica de ejercicio físico. Por otra parte, a pesar de que hemos tratado de reproducir la metodología de entrenamiento de fuerza hipertrofia desarrollada por humanos, este protocolo no refleja exactamente la metodología utilizada por los humanos para el desarrollo de la fuerza hipertrofia, por cuestiones obvias. De hecho, en humanos los niveles de estrés serían probablemente inferiores al tratarse de un comportamiento consciente, voluntario y con motivación personal. No obstante, nuestro objetivo en este estudio era profundizar en el conocimiento de la respuesta fisiológica al estrés inducida por el ejercicio de alta intensidad. En segundo lugar, la medición de algunos marcadores inmunológicos y de daño muscular tales como la inmunoglobulina A (IgA), factor de crecimiento insulínico tipo 1 (IGF-1), interleucina 1 (IL-1), IL-6, proteína quimiotáctica de monocitos-1 (MCP-1) o mioglobina, habrían sido de interés en la interpretación de los presentes resultados. Así mismo, la anestesia de los animales podría afectar a ciertos niveles plasmáticos, entre ellos la corticosterona. Por otra parte, es importante destacar que este es el primer estudio que analiza de forma global los efectos de un posible estado de sobreentrenamiento inducido por un EAI sobre índices metabólicos, marcadores plasmáticos, musculares, hepáticos y renales en un mismo trabajo, y es el primero en mostrar resultados de perfil lipídico hepático.

\section{Conclusiones}

En definitiva, podemos concluir que bajo nuestro diseño experimental hemos observado que un programa de EAI en roedores ha inducido un estado de estrés crónico contraproducente a varios niveles fisiológicos. Con esto no queremos indicar que cargas o protocolos de entrenamiento similares puedan derivar en el mismo estado de estrés-sobreentrenamiento en humanos, sino tan solo estudiar y reproducir los efectos que dicho estrés inducido por el EAI podría provocar en deportistas que sufrieran de dicho síndrome. El objetivo fue por tanto conocer mejor los efectos fisiológicos del sobreentrenamiento sobre órganos poco estudiados, con la intención de proteger al deportista y concienciar al entrenador de la importancia del correcto control y cuantificación de las cargas (Kellmann, 2010; Meeusen y col., 2013). Podemos destacar que, sumado a lo ya conocido, las alteraciones detectadas a nivel hepático y renal podrían derivar en patologías a largo plazo.

Finalmente, los autores del presente manuscrito queremos hacer hincapié en que con este estudio no criticamos la práctica de ejercicio de alta intensidad, sino un programa descontrolado del mismo. Así, podemos concluir que la práctica de ejercicio físico de alta intensidad puede llegar a resultar insana a niveles difícilmente perceptibles si ésta no es programada adecuadamente por profesionales altamente cualificados, o si el potencial estado de sobreentrenamiento no es prevenido a nivel psicológico y fisiológico por un equipo multidisciplinar. 


\section{Referencias}

Adlercreutz, H.; Harkonen, M.; Kuoppasalmi, K.; Naveri, H.; Huhtaniemi, I.; Tikkanen, H.; Remes, K.; Dessypris, A., \& Karvonen, J. (1986). Effect of training on plasma anabolic and catabolic steroid hormones and their response during physical exercise. International Journal of Sports Medicine, 7 Suppl 1, 27-28.

Aparicio, V. A.; Nebot, E.; Kapravelou, G.; Sanchez, C.; Porres, J. M.; Lopez Jurado, M., \& Aranda, P. (2011). [Resistance training reduces the metabolic acidosis and hepatic and renal hypertrophy caused by the consumption of a high protein diet in rats]. Nutrición Hospitalaria, 26(6), 1478-1486.

Aparicio, V. A.; Tassi, M.; Nebot, E.; Camiletti-Moiron, D.; Ortega, E.; Porres, J. M., \& Aranda, P. (2014). High-Intensity Exercise May Compromise Renal Morphology in Rats. International Journal of Sports Medicine, 35(08), 639-644 http://dx.doi.org/10.1055/s-0033-1354383

Benghuzzi, H.; Tucci, M.; Hughes, J.; Lyon, R., \& Adams, S. (2005). Glomerular response to adrenocortical hormone alone or in combination with selenomethionine. Biomedical Science Instrumentation, 41, 74-79.

Brooks, K., \& Carter, J. (2013). Overtraining, Exercise, and Adrenal Insufficiency. Journal of Novel Physiotherapies, 3(125).

http://doi.org/10.4172/2165-7025.1000125

Catalina-Romero, C.; Calvo, E.; Sanchez-Chaparro, M. A.; Valdivielso, P.; Sainz, J. C.; Cabrera, M.; Gonzalez-Quintela, A., \& Roman, J. (2013). The relationship between job stress and dyslipidemia. Scandinavian Journal of Public Health, 41(2),142-149. http://dx.doi.org/10.1177/1403494812470400

Czepluch, F. S.; Barres, R.; Caidahl, K.; Olieslagers, S.; Krook, A.; Rickenlund, A.; Zierath, J. R., \& Waltenberger, J. (2011). Strenuous physical exercise adversely affects monocyte chemotaxis. Thrombosis and Haemostasis, 105(1), 122-130. http://dx.doi.org/10.1160/TH10-06-0363

de Salles, B. F.; Simao, R.; Miranda, F.; Novaes Jda, S.; Lemos, A., \& Willardson, J. M. (2009). Rest interval between sets in strength training. Sports Medicine, 39(9), 765777.

http://dx.doi.org/10.2165/11315230-000000000-00000

Depke, M.; Fusch, G.; Domanska, G.; Geffers, R.; Volker, U.; Schuett, C., \& Kiank, C. (2008). Hypermetabolic syndrome as a consequence of repeated psychological stress in mice. Endocrinology, 149(6), 2714-2723. http://dx.doi.org/10.1210/en.2008-0038

Dickhout, J. G., \& Krepinsky, J. C. (2009). Endoplasmic reticulum stress and renal disease. Antioxidant and Redox Signaling, 11(9), 2341-2352. http://dx.doi.org/10.1089/ARS.2009.2705

Estoppey-Stojanovski, L. (1986). [Position of the Council of Europe on the protection of animals]. Developments in Biological Standardization, 64, 3-5. http://www.ncbi.nlm.nih.gov/pubmed/3792654

Filaire, E.; Bernain, X.; Sagnol, M., \& Lac, G. (2001). Preliminary results on mood state, salivary testosterone:cortisol ratio and team performance in a professional soccer team. European Journal of Applied Physiology, 86(2), 179-184. http://www.ncbi.nlm.nih.gov/pubmed/11822478

Folch, J.; Lees, M., \& Sloane Stanley, G. H. (1957). A simple method for the isolation and purification of total lipides from animal tissues. The Journal of Biological Chemistry, 226(1), 497-509. 
Fragala, M. S.; Kraemer, W. J.; Denegar, C. R.; Maresh, C. M.; Mastro, A. M., \& Volek, J. S. (2011). Neuroendocrine-immune interactions and responses to exercise. Sports Medicine, 41(8), 621-639.

http://dx.doi.org/10.2165/11590430-000000000-00000

Fry, A. C.; Kraemer, W. J., \& Ramsey, L. T. (1998). Pituitary-adrenal-gonadal responses to high-intensity resistance exercise overtraining. Journal Applied Physiology, 85(6), 2352-2359.

Harriss, D. J., \& Atkinson, G. (2011). Update--Ethical standards in sport and exercise science research. International Journal of Sports Medicine, 32(11), 819-821. http://dx.doi.org/10.1055/s-0031-1287829

Holt, S., \& Moore, K. (2000). Pathogenesis of renal failure in rhabdomyolysis: the role of myoglobin. Experimental Nephrology, 8(2), 72-76.

Houston, M. C.; Fazio, S.; Chilton, F. H.; Wise, D. E.; Jones, K. B.; Barringer, T. A., \& Bramlet, D. A. (2009). Nonpharmacologic treatment of dyslipidemia. Progress in Cardiovascular Disease, 52(2), 61-94.

http://dx.doi.org/10.1016/j.pcad.2009.02.002

Iglesias, P.; Carrero, J. J., \& Diez, J. J. (2012). Gonadal dysfunction in men with chronic kidney disease: clinical features, prognostic implications and therapeutic options. Journal of Nephrology, 25(1), 31-42.

Inagi, R. (2009). Endoplasmic reticulum stress in the kidney as a novel mediator of kidney injury. Nephron Experimental Nephrology, 112(1), e1-9. http://dx.doi.org/10.1159/000210573

Ishigaki, T.; Koyama, K.; Tsujita, J.; Tanaka, N.; Hori, S., \& Oku, Y. (2005). Plasma leptin levels of elite endurance runners after heavy endurance training. Journal of Physiological Anthropology and Applied Human Science, 24(6), 573-578.

Kellmann, M. (2010). Preventing overtraining in athletes in high-intensity sports and stress/recovery monitoring. Scandinavian Journal of Medicine and Science in Sports, 20 Suppl 2, 95-102. http://dx.doi.org/10.1111/j.1600-0838.2010.01192.x

Kraemer, W. J.; Fleck, S. J.; Callister, R.; Shealy, M.; Dudley, G. A.; Maresh, C. M.; Marchitelli, L.; Cruthirds, C.; Murray, T., \& Falkel, J. E. (1989). Training responses of plasma beta-endorphin, adrenocorticotropin, and cortisol. Medicine and Science in Sports and Exercise, 21(2), 146-153.

Kraemer, W. J.; Noble, B. J.; Clark, M. J., \& Culver, B. W. (1987). Physiologic responses to heavy-resistance exercise with very short rest periods. International Journal of Sports Medicine, 8(4), 247-252.

http://dx.doi.org/10.1055/s-2008-1025663

Kraemer, W. J., \& Ratamess, N. A. (2005). Hormonal responses and adaptations to resistance exercise and training. Sports Medicine, 35(4), 339-361 3544.

Kreher, J. B., \& Schwartz, J. B. (2012). Overtraining syndrome: a practical guide. Sports Health, 4(2), 128-138. http://dx.doi.org/10.1177/1941738111434406

Lehmann, M.; Foster, C., \& Keul, J. (1993). Overtraining in endurance athletes: a brief review. Medicine and Science in Sports and Exercise, 25(7), 854-862. http://www.ncbi.nlm.nih.gov/pubmed/8350709 
Lehmann, M.; Wieland, H., \& Gastmann, U. (1997). Influence of an unaccustomed increase in training volume vs intensity on performance, hematological and bloodchemical parameters in distance runners. The Journal of Sports Medicine and Physical Fitness, 37(2), 110-116.

Lira, F. S.; Rosa, J. C.; Pimentel, G. D.; Tarini, V. A.; Arida, R. M.; Faloppa, F.; Alves, E. S.; do Nascimento, C. O.; Oyama, L. M.; Seelaender, M.; de Mello, M. T., \& Santos, R. V. (2010). Inflammation and adipose tissue: effects of progressive load training in rats. Lipids in Health and Disease, 9, 109. http://dx.doi.org/10.1186/1476-511X-9-109

Liu, W. Y.; He, W., \& Li, H. (2013). Exhaustive training increases uncoupling protein 2 expression and decreases $\mathrm{Bcl}-2 / \mathrm{Bax}$ ratio in rat skeletal muscle. Oxidative Medicine and Cellular Longevity, (Volume 2013) 1-7. http://dx.doi.org/10.1155/2013/780719

Lundberg, U. Stress hormones in health and illness: the roles of work and gender. (2005). Psychoneuroendocrinology. 30(10), 1017-1021.

Martin-Cordero, L.; Garcia, J. J.; Hinchado, M. D., \& Ortega, E. (2011). The interleukin-6 and noradrenaline mediated inflammation-stress feedback mechanism is dysregulated in metabolic syndrome: effect of exercise. Cardiovascular Diabetology, 10,42 .

http://dx.doi.org/10.1186/1475-2840-10-42

Masseroli, M.; O'Valle, F.; Andujar, M.; Ramirez, C.; Gomez-Morales, M.; de Dios Luna, J.; Aguilar, M.; Aguilar, D.; Rodriguez-Puyol, M., \& Del Moral, R. G. (1998). Design and validation of a new image analysis method for automatic quantification of interstitial fibrosis and glomerular morphometry. Laboratory Investigation, 78(5), 511-522.

Matsakas, A.; Macharia, R.; Otto, A.; Elashry, M. I.; Mouisel, E.; Romanello, V.; Sartori, R.; Amthor, H.; Sandri, M.; Narkar, V., \& Patel, K. (2012). Exercise training attenuates the hypermuscular phenotype and restores skeletal muscle function in the myostatin null mouse. Experimental Physiology, 97(1), 125-140.

http://dx.doi.org/10.1113/expphysiol.2011.063008

McEwen, B. S. Protection and damage from acute and chronic stress: allostasis and allostatic overload and relevance to the pathophysiology of psychiatric disorders. (2004). Annals of the New York Academy of Sciences, 1-7. http://dx.doi.org/10.1196/annals.1314.001

Meeusen, R.; Duclos, M.; Foster, C.; Fry, A.; Gleeson, M.; Nieman, D.; Raglin, J.; Rietjens, G.; Steinacker, J., \& Urhausen, A. (2013). Prevention, diagnosis, and treatment of the overtraining syndrome: joint consensus statement of the European College of Sport Science and the American College of Sports Medicine. Medicine and Science in Sports and Exercise, 45(1), 186-205.

http://dx.doi.org/10.1249/MSS.0b013e318279a10a

Moinuddin, I., \& Leehey, D. J. (2008). A comparison of aerobic exercise and resistance training in patients with and without chronic kidney disease. Advances in Chronic Kidney Disease, 15(1), 83-96.

http://dx.doi.org/10.1053/j.ackd.2007.10.004

Oudman, I.; Clark, J. F., \& Brewster, L. M. (2013). The effect of the creatine analogue beta-guanidinopropionic acid on energy metabolism: a systematic review. PLoS One, $8(1)$, e52879.

http://dx.doi.org/10.1371/journal.pone.0052879 
Peng, C. C.; Chen, K. C.; Hsieh, C. L., \& Peng, R. Y. (2012). Swimming Exercise Prevents Fibrogenesis in Chronic Kidney Disease by Inhibiting the Myofibroblast Transdifferentiation. PLoS One, 7(6), e37388.

http://dx.doi.org/10.1371/journal.pone.0037388

Peng, C. C.; Chen, K. C.; Lu, H. Y., \& Peng, R. Y. (2012). Treadmill exercise improved adriamycin-induced nephropathy. Journal of Biological Regulators and Homeostatic Agents, 26(1), 15-28.

Pereira, B. C.; Filho, L. A.; Alves, G. F.; Pauli, J. R.; Ropelle, E. R.; Souza, C. T.; Cintra, D. E.; Saad, M. J., \& Silva, A. S. (2012). A new overtraining protocol for mice based on downhill running sessions. Clinical and Experimental Pharmacology and Physiology, 39(9), 793-798.

http://dx.doi.org/10.1111/j.1440-1681.2012.05728.x

Pinheiro-Mulder, A.; Aguila, M. B.; Bregman, R., \& Mandarim-de-Lacerda, C. A. (2010). Exercise counters diet-induced obesity, proteinuria, and structural kidney alterations in rat. Pathology - Research and Practice, 206(3), 168-173. http://dx.doi.org/10.1016/j.prp.2009.11.004

Poortmans, J. R., \& Ouchinsky, M. (2006). Glomerular filtration rate and albumin excretion after maximal exercise in aging sedentary and active men. tHE Journals of Gerontology series A: Biological Science and Medical Science, 61(11), 1181-1185.

Priya, P. H., \& Reddy, P. S. (2012). Effect of restraint stress on lead-induced male reproductive toxicity in rats. Journal Expermiental Zoology part A: Ecological Genetic and Physiology, 317(7), 455-465.

http://dx.doi.org/10.1002/jez.1738

Reeves, P. G.; Nielsen, F. H., \& Fahey, G. C., Jr. (1993). AIN-93 purified diets for laboratory rodents: final report of the American Institute of Nutrition ad hoc writing committee on the reformulation of the AIN-76A rodent diet. Journal of Nutrition, 123(11), 1939-1951.

http://www.ncbi.nlm.nih.gov/pubmed/8229312

Rosmond, R. (2005). Role of stress in the pathogenesis of the metabolic syndrome. Psychoneuroendocrinology, 30(1), 1-10.

Ruiz, J. R.; Mesa, J. L.; Mingorance, I.; Rodriguez-Cuartero, A., \& Castillo, M. J. (2004). [Sports requiring stressful physical exertion cause abnormalities in plasma lipid profile]. Revista Española de Cardiología, 57(6), 499-506.

Skenderi, K. P.; Kavouras, S. A.; Anastasiou, C. A.; Yiannakouris, N., \& Matalas, A. L. (2006). Exertional Rhabdomyolysis during a $246-\mathrm{km}$ continuous running race. Medicine and Science in Sports and Exercise, 38(6), 1054-1057. http://dx.doi.org/10.1249/01.mss.0000222831.35897.5f

Slivka, D. R.; Hailes, W. S.; Cuddy, J. S., \& Ruby, B. C. (2010). Effects of 21 days of intensified training on markers of overtraining. Journal of Strength and Conditioning Research, 24(10), 2604-2612. http://dx.doi.org/10.1519/JSC.0b013e3181e8a4eb

Smith Gd Fau - Ben-Shlomo, Y.; Ben-Shlomo Y Fau - Beswick, A.; Beswick A Fau Yarnell, J.; Yarnell J Fau - Lightman, S.; Lightman S Fau - Elwood, P., \& Elwood, P. (2005). Cortisol, testosterone, and coronary heart disease: prospective evidence from the Caerphilly study. Circulation, 112(3), 332-340.

Sweat, F.; Puchtler, H., \& Rosenthal, S. I. (1964). SIRIUS RED F3BA AS A STAIN FOR CONNECTIVE TISSUE. Archives of Pathology, 78, 69-72. 
Urhausen, A.; Gabriel, H., \& Kindermann, W. (1995). Blood hormones as markers of training stress and overtraining. Sports Medicine, 20(4), 251-276.

Wang, F. F.; Wang, Q.; Chen, Y.; Lin, Q.; Gao, H. B., \& Zhang, P. (2012). Chronic stress induces ageing-associated degeneration in rat Leydig cells. Asian Journal of Andrology, 14(4), 643-648.

http://dx.doi.org/10.1038/aja.2011.183

Warren, J. D.; Blumbergs, P. C., \& Thompson, P. D. (2002). Rhabdomyolysis: a review. Muscle and Nerve, 25(3), 332-347. http://dx.doi.org/10.1002/mus.10053

Wu GI Fau - Chen, Y. S.; Chen Ys Fau - Huang, X. D.; Huang Xd Fau - Zhang, L. X., \& Zhang, L. X. (2012). Exhaustive swimming exercise related kidney injury in rats protective effects of acetylbritannilactone. International Journal of Sports Medicine, $33(1), 1-7$.

http://dx.doi.org/10.1055/s-0031-1284397

Xiao, W.; Chen, P., \& Dong, J. (2012). Effects of overtraining on skeletal muscle growth and gene expression. International Journal of Sports Medicine, 33(10), 846-853. http://dx.doi.org/10.1055/s-0032-1311585 